\title{
Assessing Knowledge of Hypoglycemia Symptoms among Type 2 Diabetic Patients Using Insulin, Khartoum, Sudan
}

\author{
Abdelhaleem Mustafa Madani', Asma Noureldaim Mahmoud2, \\ Hisham Mohammed Abdelrahim³ ${ }^{3}$ Tarig Mohammed El-Hadiyah", Abubakr Khidir Yousif ${ }^{*}$ \\ ${ }^{1}$ Pharmacology Department, Faculty of Pharmacy, International University of Africa, Khartoum, Sudan \\ ${ }^{2}$ Department of Pharmacy Practice, Faculty of Pharmacy, International University of Africa, Khartoum, Sudan \\ ${ }^{3}$ University of National Ribat, Khartoum, Sudan \\ ${ }^{4}$ Department of Pharmacology, Faculty of Pharmacy, International University of Africa, Khartoum, Sudan \\ ${ }^{5}$ Ministry of Health, Wadmadani City, Sudan \\ Email: helmi7011@gmail.com, *abubarkhidir@hotmail.com
}

How to cite this paper: Madani, A.M., Mahmoud, A.N., Abdelrahim, H.M., El-Hadiyah, T.M. and Yousif, A.K. (2019) Assessing Knowledge of Hypoglycemia Symptoms among Type 2 Diabetic Patients Using Insulin, Khartoum, Sudan. Pharmacology \& Pharmacy, 10, 21-30.

https://doi.org/10.4236/pp.2019.101003

Received: November 28, 2018

Accepted: January 14, 2019

Published: January 17, 2019

Copyright $\odot 2019$ by author(s) and Scientific Research Publishing Inc. This work is licensed under the Creative Commons Attribution International License (CC BY 4.0).

http://creativecommons.org/licenses/by/4.0/

\begin{abstract}
Background: Hypoglycemia is a traumatic dilemma that has a critical impact on not only the patient's quality of life but also their families and communities. Improving Patient's awareness and knowledge regarding hypoglycemia symptoms is crucial to avoid serious implications like loss of consciousness and even death. Objective: This study aimed to assess patients' knowledge of hypoglycemia symptoms and source of diabetes information among insulin users type 2 diabetes. Methods: A single centre, hospital-based, analytical, and cross-sectional study was carried out among diabetic patients using insulin attended to Ribat University Hospital Diabetes Clinic (RUHDC), Khartoum State, Sudan during a period of study. A validated questionnaire was used to obtain socio-demographic characteristics of respondents and the patients' knowledge regarding hypoglycemia symptoms was assessed whether good, medium or poor. Data were analyzed, using (SPSS). Descriptive (frequencies) and inferential statistics (association used chi-square) were used at a level of confidence $p \leq 0.05$. Results: A majority of patients (52\%) had poor knowledge of hypoglycemia symptoms and the common symptoms known to patients were hands tremor (67.2\%), blurred vision (52.8\%) and sweating (65.6\%). No associations were found among hypoglycemia symptoms knowledge, socio-demographic and diabetes duration. Only (25.6\%) had regular visiting to educating center and physicians were the major source of diabetes knowledge (74.4\%). Conclusion: This study proved low patients hypoglycemia symptoms knowledge and physician were the main sources of informa-
\end{abstract}


tion about diabetes at RUHDC. Further ideas are required in collaboration and integrated approach to filling the gap of patients' awareness and multidisciplinary team in diabetes management.

\section{Keywords}

Hypoglycemia Symptoms, Knowledge, Type 2 Diabetes, Insulin-Users, Sudan

\section{Introduction}

Hypoglycemia is a true medical emergency [1] and has a critical effect on mortality, morbidity, and quality of life [2]. Hypoglycemia is a major barrier for attaining treatment goals and reduction of long-term complications in diabetes management and therefore it is an important reason for increased costs of medical care [3].

The American Diabetes Association defines the hypoglycemia as "any abnormally low plasma glucose concentration that exposes the subject to potential harm", and proposes a threshold of $<70 \mathrm{mg} / \mathrm{dl}$ [4]. The spectrum of symptoms depends on the duration and severity of hypoglycemia and varies from autonomic activation to behavioral changes to altered cognitive function to seizures or coma and even death [5].

Two types of hypoglycemia symptoms are documented. The first one is neuroglycopenia which causes symptoms such as blurred vision, fatigue, concentrating disturbance, confusion and behavioral changes, and may lead to loss of consciousness, seizures, brain damage, and death [6] [7]. The second one is neurogenic symptoms and this occurs by the hormones and neurotransmitters delivered as a result of low brain glucose levels, causing symptoms such as tremor, palpitation, anxiety, sweating, hunger, dizziness and drowsiness [7] [8].

Patient's awareness and knowledge of every hypoglycemia symptoms are very important to be early recognized and to take measures for treatment and avoid risks that may even cause death [7].

Many studies [9] [10] report that younger diabetes patients have high knowledge about diabetes in general and this is because older have a low cognitive function which hinders diabetes education programs, whilst younger ages have higher motivation [11] and adaptability towards disease.

Higher educated patients usually have higher knowledge about diabetes and this is attributed to the fact that educated patients can easily respond to education programs and have the ability to communicate with other sources of knowledge that provides medical programs such as television, radio, and internet [11].

Diabetes management is a complex and cost-effective process and requires an integrated multi-disciplinary team consisting of physicians, nurses, dietitians, exercise specialists, pharmacists, dentists, podiatrists, and mental health professionals, however, unless the presence of educated patients who are able to care about themselves. The efforts of this team will not provide effective care. Partic- 
ipation of diabetic patients in diabetes self-management education (DSME) is an essential element of diabetes care [12]. Education helps people with diabetes to initiate effective self-management and cope with diabetes when they are first diagnosed. DSME helps patients to optimize metabolic control, prevent and manage complications, and maximize the quality of life in a cost-effective manner [13].

In the past few decades, a great shift has occurred regarding tradition clinical pharmacists role, as medications dispenser, to direct patients' pharmaceutical care such as diabetes management [14] [15] including diabetes patients' education [16].

Knowledge and awareness of symptoms of hypoglycemia in diabetes prevent patients from many risks of diabetes; however, the presence of studies that check patient's knowledge about this important part of diabetes self-management is scarce and fragmented in Sudan.

\section{Methods}

\subsection{Patients Characteristics}

All type-2 diabetic patients on at least three-month insulin treatment attended to Ribat University Hospital Diabetes Clinic (RUHDC) during the period of $\mathrm{Au}$ gust 2012 to February 2013 were recruited in this study, except those who refused to participate (just two patients).

\subsection{Instrument of Data Collection}

Self-administered questionnaire constructed from recent literature and validated by a discussion with experts group in diabetes management at Ribat University. Then the questionnaire was piloted randomly to ten diabetic patients. After minor changes, the questionnaire distributed directly to all selected sample, whilst patients' cards were also used to obtain some patients data.

\subsection{Hypoglycemia Symptoms Knowledge Assessment}

Information including socio-demographic characteristics and the patients' knowledge of hypoglycemia symptoms was assessed by using closed questions (yes or no). Symptoms variables included in the questionnaire were dizziness, weakness, drowsiness, hands tremor, sweating, palpitation, excessive hunger, and blurred vision. Patients able to identify more than four hypoglycemia symptoms in the questionnaire considered having good knowledge, those identified four were medium and those identified less than four had poor knowledge. Patients also were asked about the source of knowledge of diabetes and their regular visiting to diabetes educating team at RUHDC.

\subsection{Data Analysis}

Data analyzed, using social package for social science (SPSS) version 16, to assess patient's answers. Descriptive (frequencies) and inferential statistics (association 
used chi-square) were used at a level of confidence $\mathrm{p} \leq 0.05$.

\section{Results}

A total of 125 respondents recruited in this study, 96 (76.8\%) were females. Age of participants between 41 and 60 years were 65 (52\%), patients with basic education level were $53(42.4 \%)$ and $80(64 \%)$ had low financial status. Majority of patients, (52\%) had poor hypoglycemia symptoms knowledge No associations were found between hypoglycemia symptoms knowledge, socio-demographic and diabetes duration (Table 1 demonstrated the Association between Socio-Demographic variables and patients' hypoglycemia knowledge). The common symptoms known to patients were hands tremor (67.2\%), blurred vision (52.8\%) and sweating (65.6\%), see Table 2 . The hypoglycemia symptoms were interpreted to three

Table 1. Association between patients' hypoglycemia knowledge and some variables.

\begin{tabular}{|c|c|c|c|c|}
\hline \multirow{3}{*}{ Socio-demographic } & \multicolumn{3}{|c|}{ Hypoglycemia knowledge } & \multirow{3}{*}{$\mathrm{p}$ value } \\
\hline & \multicolumn{3}{|c|}{$\mathrm{N}=(\%)$} & \\
\hline & Good & Medium & Poor & \\
\hline $1-\operatorname{Sex}$ & & & & 0.737 \\
\hline Male & $13(10.4)$ & $1(0.8)$ & $15(12)$ & \\
\hline Female & $39(13.2)$ & $7(5.6)$ & $50(40)$ & \\
\hline 2-Age & & & & 0.718 \\
\hline $20-40$ & $4(3.2)$ & $0(0.0)$ & $4(3.2)$ & \\
\hline $41-60$ & $26(20.8)$ & $6(4.8)$ & $33(26.4)$ & \\
\hline Above 60 & $22(17.6)$ & $2(1.6)$ & $28(22.4)$ & \\
\hline 3-Educational level & & & & 0.086 \\
\hline Basic & $20(16)$ & $2(1.6)$ & $31(24.8)$ & \\
\hline High secondary & $9(7.2)$ & $2(1.6)$ & $13(10.4)$ & \\
\hline University & $11(8.8)$ & $1(0.8))$ & $4(3.2)$ & \\
\hline Non-formal & $1(0.8)$ & $1(0.8)$ & $0.0(0.0)$ & \\
\hline Illiterate & $11(8.8)$ & $2(1.6)$ & $17(13.6)$ & \\
\hline 4-Financial status & & & & 0.354 \\
\hline High & $20(16)$ & $1(0.8)$ & $24(19.2)$ & \\
\hline Low & $32(25.6)$ & $7(5.6)$ & $41(32.8)$ & \\
\hline 5-Diabetes duration & & & & 0.263 \\
\hline $1-5$ & $8(6.4)$ & $1(0.8)$ & $8(6.4)$ & \\
\hline $6-10$ & $7(5.6)$ & $14(11.2)$ & $4(3.2)$ & \\
\hline $11-15$ & $15(12)$ & $2(1.6))$ & $20(16)$ & \\
\hline $16-20$ & $5(4)$ & $0(0.0)$ & $8(6.4)$ & \\
\hline $21-25$ & $2(1.6)$ & $1(0.8)$ & $11(8.8)$ & \\
\hline Above 25 & $8(6.4)$ & $0(0.0)$ & $3(2.4)$ & \\
\hline
\end{tabular}


categories poor, medium and good (see Figure 1). The physicians were the major source of hypoglycemia knowledge for patients (74.4\%), see Figure 2. Only (25.6\%) had regular visiting to educating. center (see Figure 3 ).

\section{Discussion}

This study was carried out among 125 type 2 diabetes attending RUHDC and more than half of the patients in this study had poor hypoglycemia symptoms knowledge and these findings was less than reported by Almalki et al. [17] who reported $85 \%$ of patients had sufficient knowledge about the symptoms of hyperglycemia and this high percent is attributed to the fact that more than half of his patients received information about diabetes management from medical

Table 2. Knowledge of symptoms of hypoglycemia among insulin users type 2 DM patients.

\begin{tabular}{ccc}
\hline Symptoms & Symptoms knowledge of hypoglycemia frequency & Percent $(\%)$ \\
\hline Hands tremor & 84 & 67.2 \\
Blurred vision & 66 & 52.8 \\
Sweating & 82 & 65.6 \\
Drowsiness & 53 & 42.4 \\
Dizziness & 50 & 40 \\
Palpitation & 38 & 30.4 \\
Excessive hunger & 10 & 8 \\
Fatigue & 7 & 5.6 \\
\hline
\end{tabular}

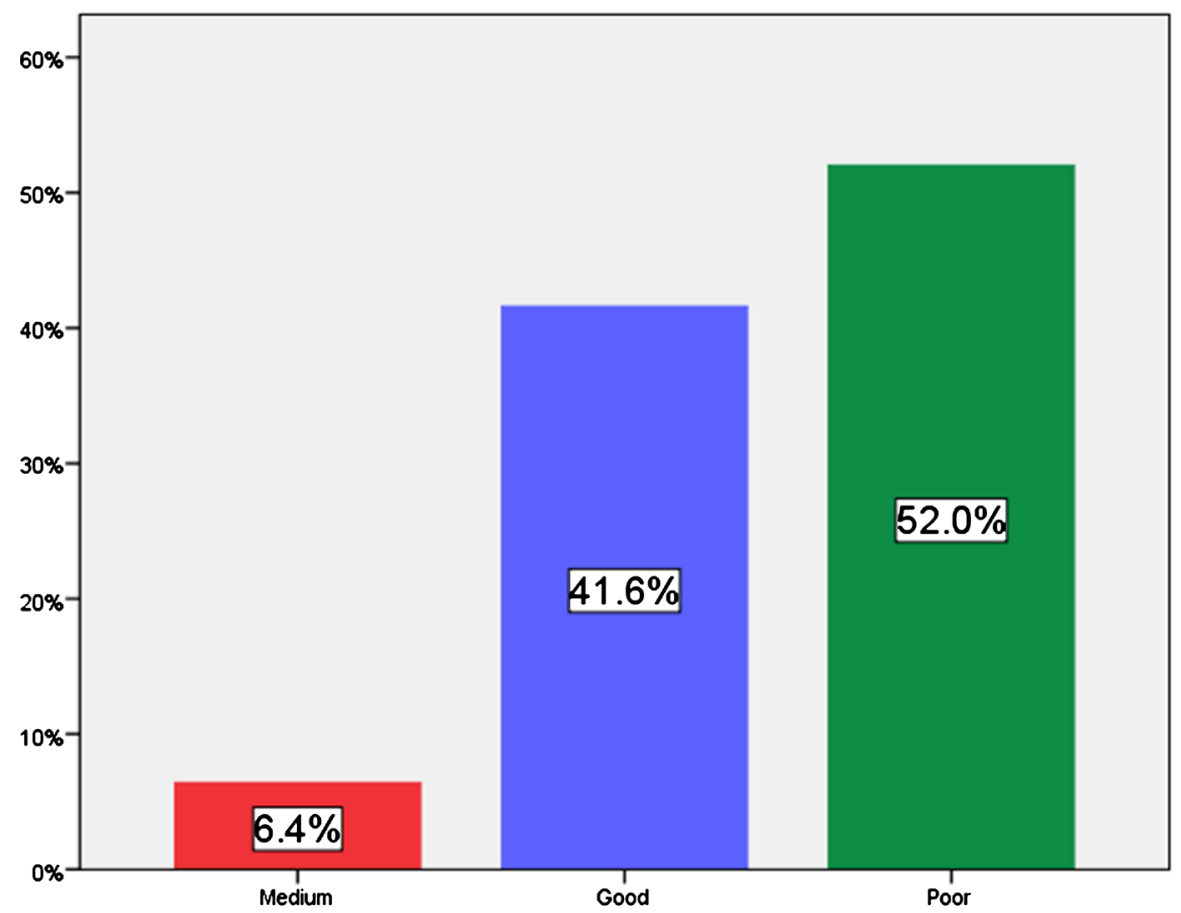

Figure 1. Interpretation of patients knowledge about hypoglycemia symptoms. 


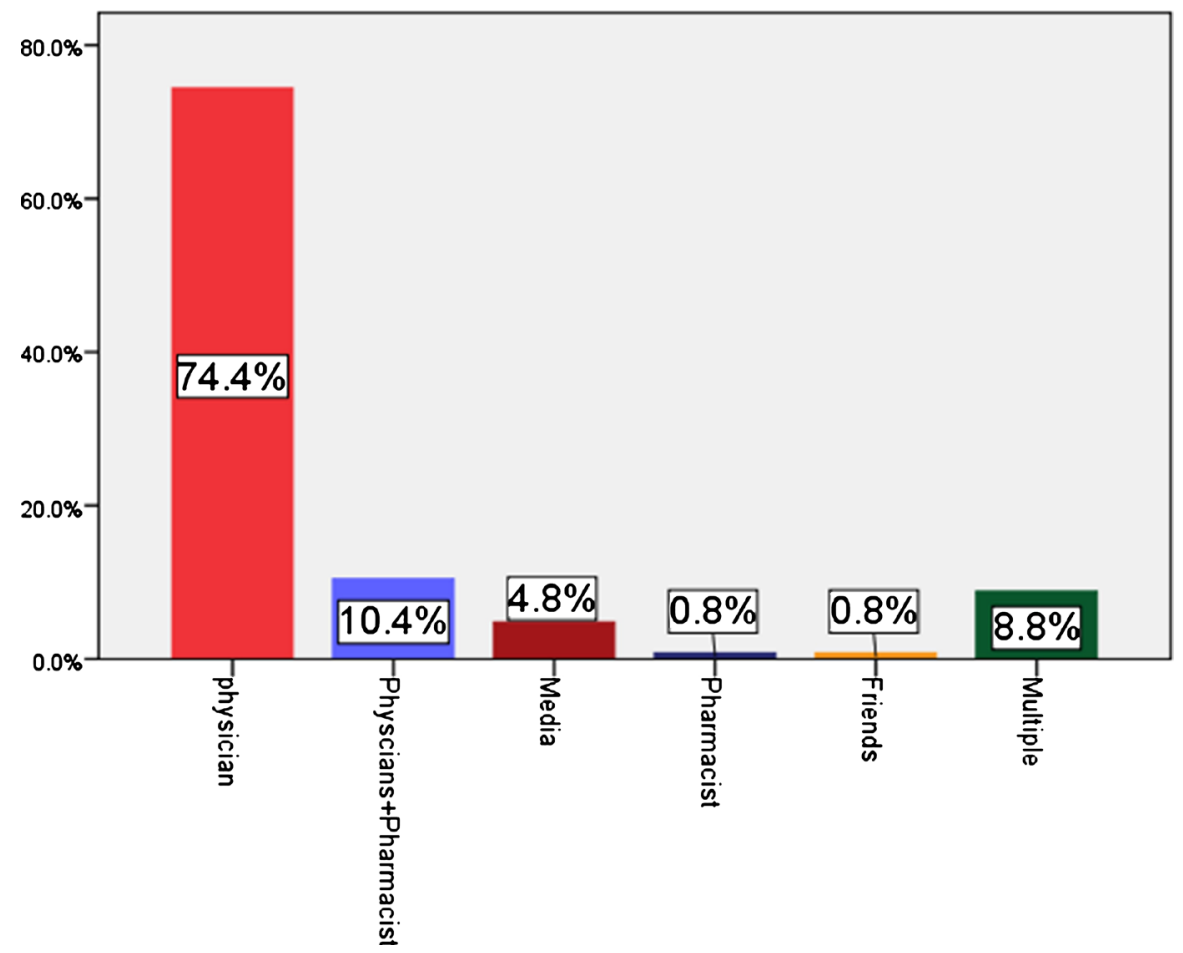

Figure 2. Patients source of information about diabetes.

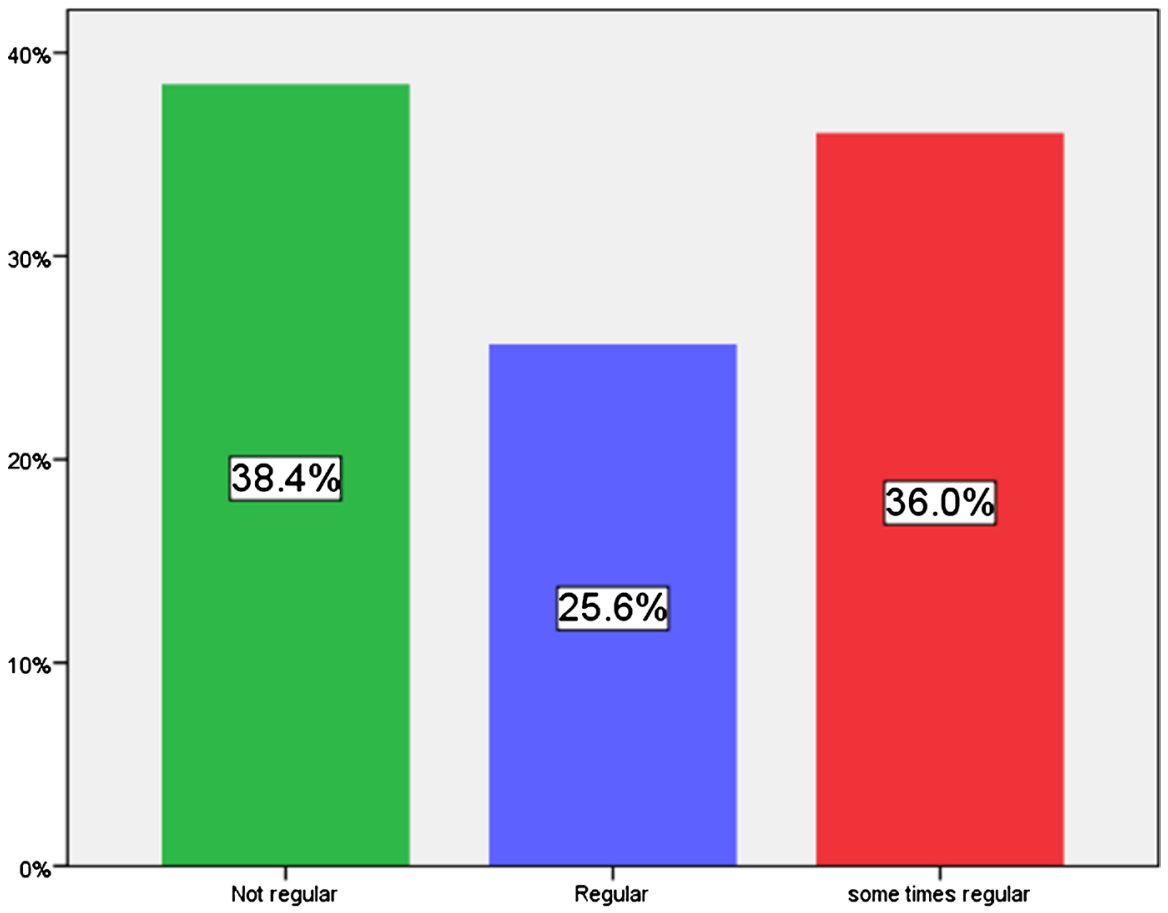

Figure 3. Patients' commitment to regular visit to diabetes educating team.

providers. On the other hand, Kazi and his colleagues [18] reported only $17.2 \%$, whilst El-Khawaga from Egypt reported 48.1\% [19].

Older patients with type 2 diabetes have a higher risk of developing a severe episode of hypoglycemia due to low cognitive function [20]. Comprehensive 
evaluations for this group of patients, including assessment of their knowledge about symptoms of hypoglycemia, can help in preventing the risk of hypoglycemia. This study did not reveal an association between age and knowledge about hypoglycemia symptoms and this finding contradicted with that reported by Thomson and his colleagues [9].

No association was found between hypoglycemia symptoms knowledge and sex in this study and this consistent with Herath and his colloquies who revealed no significant relationship between sex and diabetes knowledge [21]. On the other hand, this result reflects another picture that different from Awad study who reported that Sudanese female usually cope less well with diabetes, this may be due to equality in the chance of education between male and female in Sudan during last decades [22].

Usually, diabetic patients with high education level have good knowledge score about diabetes [23], diabetes risk factors [24] and diabetes self-management however education level showed no effect on hypoglycemia symptoms knowledge in this study. This result was consistent with Chinnappan and his colleagues who revealed no relationship between education level and patients diabetes knowledge [25].

Although high relation between patients' diabetes self-management knowledge and diabetes duration was found [26], however, no significant relationship was found between diabetes duration and hypoglycemia symptoms knowledge in this study and this consistent with that reported by Shriraam study [7].

Regarding hypoglycemia symptoms knowledge, sweating, tremor of hand and blurred vision were the most symptoms identified by our patients in this study, whilst Shriraam [7] reported that most common symptoms known to his patients were dizziness (81.4\%), weakness (73.8\%), and drowsiness (72.1\%).

Diabetes treatment is complex and multidisciplinary team expert in diabetes management can provide effective health care service, including diabetes self-management knowledge. The ideal team may consist of physicians, nurse practitioners, physician assistants, nurses, dietitians, exercise specialists, pharmacists, dentists, podiatrists, and mental health professionals [27].

Almost three-quarter of patients in this study revealed that physicians were the main source of diabetes knowledge, whilst pharmacist reported only minor role. This finding consisted with Al-Maskari [28] who reported 2.1\% for pharmacist as a source of diabetes knowledge. This week role of the pharmacist in addition to the poor commitment of patient to a regular visit to diabetes educating team gave justification for low percent of hypoglycemia symptoms knowledge in this study.

\section{Conclusion}

This study proved low patients hypoglycemia symptoms knowledge and the physicians were the main source of information about diabetes at RUHDC, and this subsequently increased the burden on physicians and decrease the effective- 
ness of diabetes management. Great efforts are required as collaboration and integrated multidisciplinary team, including effective participation of the clinical pharmacist, to fill the gap of the patients' awareness of complex diabetes management.

\section{Limitation}

Although RUHDC is one of the largest centers in Sudan, conducting this study in a single centre will not give the complete picture about patients' knowledge of hypoglycemia symptoms and source of diabetes information among insulin users type 2 diabetes in Sudan as a whole, so generalized study funded by considerable organization or research institute is strongly recommended.

\section{Ethics Approval and Consent to Participate}

Ethical approval to the study from Higher Graduated Studies, University of Ribat was issued. All participants were informed verbally about the aims of the study and gave chance to participate or refuse.

\section{Authors' Contributions}

The first Author planned, collected, analyzed data, findings and wrote the paper. While the others were deeply involved in all steps as supervisors to the study from proposal up to writing manuscript, revising and approval of the final version.

\section{Acknowledgements}

The Authors appreciated the RUHDC workers, doctors and patients for their kind participation, response, and patience during the period of the study.

\section{Funding}

This study was not funded by a grant from any organization. It is original work of the First Author as Research for M Sc Degree in clinical pharmacy.

\section{Conflicts of Interest}

The authors declare that they have no competing interests.

\section{References}

[1] Shafiee, G., et al. (2012) The Importance of Hypoglycemia in Diabetic Patients. Journal of Diabetes \& Metabolic Disorders, 11, 17. https://doi.org/10.1186/2251-6581-11-17

[2] Bruce, D., et al. (2009) Severe Hypoglycaemia and Cognitive Impairment in Older Patients with Diabetes: The Fremantle Diabetes Study. Diabetologia, 52, 1808. https://doi.org/10.1007/s00125-009-1437-1

[3] Bhutani, G., et al. (2015) Effect of Diabetic Education on the Knowledge, Attitude and Practices of Diabetic Patients towards Prevention of Hypoglycemia. Indian Journal of Endocrinology and Metabolism, 19, 383. 
https://doi.org/10.4103/2230-8210.152781

[4] Association, A.D. (2018) 6. Glycemic Targets: Standards of Medical Care in Diabetes-2018. Diabetes Care, 41, S55-S64. https://doi.org/10.2337/dc18-S006

[5] Ejegi, A., Ross, A.J. and Naidoo, K. (2016) Knowledge of Symptoms and Self-Management of Hypoglycaemia amongst Patients Attending a Diabetic Clinic at a Regional Hospital in KwaZulu-Natal. African Journal of Primary Health Care \& Family Medicine, 8, 1-6. https://doi.org/10.4102/phcfm.v8i1.906

[6] Towler, D.A., et al. (1993) Mechanism of Awareness of Hypoglycemia: Perception of Neurogenic (Predominantly Cholinergic) Rather than Neuroglycopenic Symptoms. Diabetes, 42, 1791-1798. https://doi.org/10.2337/diab.42.12.1791

[7] Shriraam, V., et al. (2015) Knowledge of Hypoglycemia and Its Associated Factors among Type 2 Diabetes Mellitus Patients in a Tertiary Care Hospital in South India. Indian Journal of Endocrinology and Metabolism, 19, 378. https://doi.org/10.4103/2230-8210.152779

[8] Cefalu, C.A. and Cefalu, W.T. (2005) Controlling Hypoglycemia in Type 2 Diabetes: Which Agent for Which Patient? At Each New Stage of Treatment, Choices Can Be Made to Reduce Risk. Journal of Family Practice, 54, 855-863.

[9] Thomson, F., et al. (1991) Lack of Knowledge of Symptoms of Hypoglycaemia by Elderly Diabetic Patients. Age and Ageing, 20, 404-406. https://doi.org/10.1093/ageing/20.6.404

[10] Mutch, W. and Dingwall-Fordycet, I. (1985) Is It a Hypo? Knowledge of the Symptoms of Hypoglycaemia in Elderly Diabetic Patients. Diabetic Medicine, 2, 54-56. https://doi.org/10.1111/j.1464-5491.1985.tb00593.x

[11] Shrestha, N., et al. (2015) Diabetes Knowledge and Associated Factors among Diabetes Patients in Central Nepal. International Journal of Collaborative Research on Internal Medicine \& Public Health, 7, 82.

[12] Mulcahy, K., et al. (2003) Diabetes Self-Management Education Core Outcomes Measures. The Diabetes Educator, 29, 768-803. https://doi.org/10.1177/014572170302900509

[13] Nicholas, D., et al. (2001) Digital Health Information Provision and Health Outcomes. Journal of Information Science, 27, 265-276. https://doi.org/10.1177/016555150102700409

[14] Chisholm-Burns, M.A., et al. (2010) US Pharmacists' Effect as Team Members on Patient Care: Systematic Review and Meta-Analyses. Medical Care, 923-933. https://doi.org/10.1097/MLR.0b013e3181e57962

[15] Simpson, S.H., et al. (2011) Effect of Adding Pharmacists to Primary Care Teams on Blood Pressure Control in Patients with Type 2 Diabetes: A Randomized Controlled Trial. Diabetes Care, 34, 20-26. https://doi.org/10.2337/dc10-1294

[16] Farsaei, S., et al. (2011) Effect of Pharmacist-Led Patient Education on Glycemic Control of Type 2 Diabetics: A Randomized Controlled Trial. Journal of Research in Medical Sciences: The Official Journal of Isfahan University of Medical Sciences, 16, 43.

[17] Almalki, M.H., et al. (2018) Assessment of Ramadan Education and Knowledge among Diabetic Patients. Clinical Medicine Insights. Endocrinology and Diabetes, 11. https://doi.org/10.1177/1179551417751611

[18] Kazi, R.N., Bote, M.M. and Raikar, K.J. (2017) Knowledge, Attitude and Practices about Diabetes Mellitus and Its Complications in T2DM Patients Attending the UHC in Mumbai. International Journal of Community Medicine and Public Health, 4, 2793-2797. https://doi.org/10.18203/2394-6040.ijcmph20173325 
[19] El-Khawaga, G. and Abdel-Wahab, F. (2015) Knowledge, Attitudes, Practice and Compliance of Diabetic Patients in Dakahlia, Egypt. European Journal of Medical Research, 3, 40-53.

[20] Punthakee, Z., et al. (2012) Poor Cognitive Function and Risk of Severe Hypoglycemia in Type 2 Diabetes: Post Hoc Epidemiologic Analysis of the ACCORD Trial. Diabetes Care, 35, 787-793.

[21] Herath, H.M., et al. (2017) Knowledge, Attitude and Practice Related to Diabetes Mellitus among the General Public in Galle District in Southern Sri Lanka: A Pilot Study. BMC Public Health, 17, 535. https://doi.org/10.1186/s12889-017-4459-5

[22] Mohamed Ahmed, A. and Hassan Ahmed, N. (2001) Diabetes Mellitus in Sudan: The Size of the Problem and the Possibilities of Efficient Care. Practical Diabetes International, 18, 324-327. https://doi.org/10.1002/pdi.292

[23] Al-Adsani, A., et al. (2009) The Level and Determinants of Diabetes Knowledge in Kuwaiti Adults with Type 2 Diabetes. Diabetes \& Metabolism, 35, 121-128. https://doi.org/10.1016/j.diabet.2008.09.005

[24] Aljoudi, A.S. and Taha, A.Z. (2009) Knowledge of Diabetes Risk Factors and Preventive Measures among Attendees of a Primary Care Center in Eastern Saudi Arabia. Annals of Saudi Medicine, 29, 15. https://doi.org/10.4103/0256-4947.51813

[25] Chinnappan, S., et al. (2017) Assessment of Knowledge of Diabetes Mellitus in the Urban Areas of Klang District, Malaysia. Pharmacy, 5, 11.

https://doi.org/10.3390/pharmacy5010011

[26] Ko, S.-H., et al. (2012) Influence of the Duration of Diabetes on the Outcome of a Diabetes Self-Management Education Program. Diabetes \& Metabolism Journal, 36, 222-229. https://doi.org/10.4093/dmj.2012.36.3.222

[27] McGill, M., et al. (2017) The Interdisciplinary Team in Type 2 Diabetes Management: Challenges and Best Practice Solutions from Real-World Scenarios. Journal of Clinical \& Translational Endocrinology, 7, 21-27. https://doi.org/10.1016/j.jcte.2016.12.001

[28] Al-Maskari, F., et al. (2013) Knowledge, Attitude and Practices of Diabetic Patients in the United Arab Emirates. PLOS ONE, 8, e52857. https://doi.org/10.1371/journal.pone.0052857 